\title{
Parameters Optimization of Region growing Segmentation Based on Differential Evolution algorithm
}

\author{
Wanli Huang ${ }^{1, a}$ \\ ${ }^{1}$ College of Geographical Sciences, Fujian Normal University, Fuzhou, 350007, China \\ aemail: hwl77cn@163.com
}

Keywords: Region Growing; Image Segmentation; Parameters Optimization; Differential Evolution

\begin{abstract}
Image segmentation is the first step of GEOgraphical Based Image Analysis (GEOBIA). Because of no obvious mathematical relationship between the parameters of segmentation algorithm and the optimal segments, users select parameters by "trial and error" method, which is time-consuming and subjective. This paper used the differential evolution algorithm to automatically optimize the parameters of region growing algorithm. Because of differential evolution algorithm is a heuristic optimization method, use this method to select the parameters of segmentation for optimal segments is objective and efficient. The experiment result shown that use this method to select the optimal segmentation parameters can get the accuracy result and improve the efficiency.
\end{abstract}

\section{Introduction}

In recent years, spatial resolution of remote sensing image is improved substantially. As a result, texture and geometrical shape of objects in the image are clearer and finer. At the same time, the corresponding image of ground object becomes more complicated. There is a great challenge for how to extract meaningful information from these images of high spatial resolution. As an effective method of analyzing remote sensing image of high spatial resolution, geographic object-based image analysis (GEOBIA) has established a bridge between remote sensing image of high spatial resolution and GIS, and it develops rapidly in remote sensing during recent years. As a primary and key step of GEOBIA, image segmentation directly affects accuracy of the follow-up analysis and classification.

The region growing algorithm proposed by Baatz and Schäpe is the most popular segmentation method for remote sensing images [1]. This algorithm has three main parameters: the scale, the weight of color, and the weight of compactness. The scale parameter controls indirectly the size of the final segments, and the weights of color and compactness impact the shape of the final segments. In order to get the optimal segments, we must give the optimal parameters values.

The objects on remote sensing image are complicated and variable; moreover, scale parameters of segmentation algorithm do not have an explicit meaning, it is difficult to establish explicit relations with geographical object scale, and it is hard to set up an objective and effective method of selecting the optimal segmentation scale parameter. Under this situation, users often choose an "optimal" segmentation by carrying out a series of segmentation for the image. The problem about parameters selection for segmentation can be solved to some extent via the subjective and qualitative parameters selection method, "trial and error", by relying on human visual system [2] [3]. However, this method is time-consuming, strenuous and subjective, and it is difficult to accurately acquire the optimal segmentation parameters. Therefore, an objective and qualitative method is required to select the optimal segmentation parameters in research and application, so as to gain an optimal result.

At present, the quantitative method for selecting the "optimal" segmentation result and parameters is experimental evaluation method of segmentation assessment. The appropriate segmentation parameters were chosen by applying supervised and unsupervised evaluation of goodness value [4]. Unsupervised selection method uses the goodness value as the judgment and selects the segmentation result and parameters corresponding to the optimal goodness value as the 
“optimal" segmentation result and parameters by calculating the goodness values of a series of segmentation results [5]-[8]. This method get the fitness value based on the all image segments, so the optimal parameters are only suitable for the dominating objects in the image. It is hard to get corresponding “optimal” segmentation parameters of different class objects. Supervised evaluation tries to select a relatively optimal segmentation result by comparing a series of segmentation results and reference polygons; meanwhile, the corresponding parameters are selected as the "optimal" segmentation parameters. This method can draw reference polygons of specific class objects and the optimal parameters are suitable for segmenting the specific class objects.

One key factor of affecting segmentation parameters selection via supervised evaluation is the measure of fitness. The fitness should reflect the optimal segmentation or find out the over-segmentation and under-segmentation problems [9]. Another key factor is whether the segmentation parameters were the optimal. The parameters of segmentation have infinite variety within their ranges, and the usual methods are to exhaust the values in a certain value interval and scope. Later, the fitness between the segments and the reference polygons are calculated, and the value with the smallest discrepancy will be selected as the optimal segments [9][10]. Such method has great blindness in measuring fitness and selecting the optimal parameters. Except the large amount of calculation, there might be a difference between the calculated optimal parameters and the real optimal parameters if interval of parameters is not set properly.

As a heuristic optimization method, differential evolution (abbrev DE) algorithm is the self-organizing and self-adaptive artificial intelligence technology. It is a parallel random search algorithm proposed by the American scholars Storn and Prince [11]. Different from other evolution algorithms (such as genetic algorithm and particle swarm optimization), differential evolution algorithm has retained global search strategy based on population. By adopting real-number encoding, simple mutation operation based on difference, and one-to-one competition survival strategy, it has reduced complexity of evolution operation. Due to its evolution operation as well as strong global convergence ability and robustness, it is very suitable for solving some optimization problems in complex environment. In this paper, differential evolution algorithm is introduced into image segmentation parameters selection via supervised evaluation. By selecting the optimal segmentation parameters according to reference polygons and fitness criterion, the blindness of segmentation parameters selection can be reduced.

\section{The Design of Parameters Optimization Based on Differential Evolution Algorithm}

Figure 1 shows the workflow of the optimal parameters selection for region growing based on differential evolution algorithm according to the reference polygons and fitness criterion.

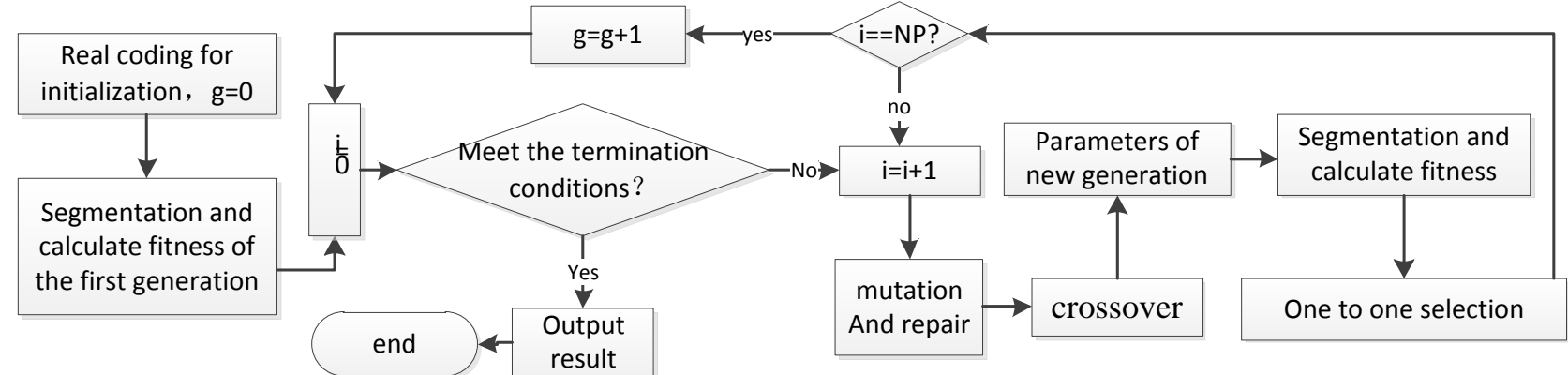

Fig.1. The workflow of parameters optimization based on DE method

\section{(1) Real coding and population initialization}

Differential evolution algorithm applies real encoding. The Baatz region growing segmentation algorithm adopted in this paper has 3 segmentation parameters which are scale parameter, weight of color and weight of compactness. So problem solving of differential evolution is three-dimensional. The initial population can be expressed as: $\left\{x_{i}(0) \mid x_{i, j}^{L} \leq x_{i, j}(0) \leq x_{i, j}^{U}\right\}$. The generation formula of the population is $x_{i, j}(0)=x_{i, j}^{L}+\operatorname{rand}(0,1)\left(x_{i, j}^{U}-x_{i, j}^{L}\right), i=1,2, \ldots, N P ; j=1,2, \ldots, D$. Where $N P$ 
is the number of population, and it is the input parameter of evolution algorithm; $\mathrm{D}$ is the dimension of problem solving and its value is 3 in this segmentation method; $x_{i, j}(0)$ is the component of population $i$ at dimension $j$; $x_{i, j}^{L}$ and $x_{i, j}^{U}$ are the minimum and maximum value ranges of component at dimension $j$, it refers to the search scope of segmentation scale or weight of this segmentation algorithm, and it will be set as the scope of selecting the optimal parameters input by the user; rand $(0,1)$ is random function and its value ranges $[0,1]$.

\section{(2) Calculation of fitness}

Image segmentation is conducted for all segmentation parameters corresponding to the individuals in the current generation, to gain the segments of each population. Meanwhile, the fitness value between each individual segments and reference polygons is calculated according to the measure of fitness. In this study, Precision and Recall $(F)$ fitness is adopted as a measure [12]. This fitness uses the four difference regions to calculate the fitness according to different overlapping situations of reference polygons and segments. The definitions of the four regions was shown in Figure 2. The region encircled by green lines means the reference polygon $R$, and the region encircled by red lines indicates the segment region $S$; the region $t p$ is the area of correct segmentation ( $t p=R \cap S)$, the $f p$ region is the area of over-segmentation ( $f p=S-(R \cap S)$ ), the region $f n$ means the area of under-segmentation $(f n=R-(R \cap S))$, and the remaining part refers to irrelevant area, denoted with $t n$ ( $t n=I-(R \cup S), I$ is the whole image). According to such definition of region, correct segmentation rate $R_{s k}$ based on segment and correct segmentation rate $R_{R k}$ based on reference polygon can be defined. And they can be calculated according to Equation (1), and the measurement $F_{k}$ of fitness can be calculated according to Equation (2). The fitness $F$ of all segments corresponding to the reference polygons can be calculated according to Equation (3).

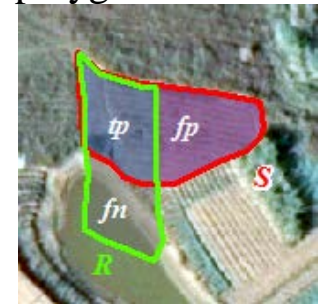

Fig.2. The four difference regions of image

$$
\begin{aligned}
& R_{s k}=\frac{t p_{k}}{t p_{k}+f p_{k k}}, R_{r k}=\frac{t p_{k}}{t p_{k}+f n_{k}} \\
& F_{k}=1-\frac{2 R_{s k} R_{r k}}{R_{s k}+R_{r k}} \\
& F=\frac{1}{N_{r}} \sum_{k=1}^{N_{r}}\left(1-\frac{2 R_{s k} R_{r k}}{R_{s k}+R_{r k}}\right)
\end{aligned}
$$

\section{(3) Judgment for termination}

Termination conditions of $\mathrm{DE}$ are as follows: the evolution generation reaches the maximum value ( $g \geq G, G$ is the maximum generation of $D E$ ) or the absolute value of the difference value between the fitness value of the optimal individual in the current generation of population and the value of the optimal individual in the last generation of population is smaller than the threshold of error $\left(\left|f_{g}(\cdot)-f_{g-1}(\cdot)\right| \leq t h_{f}\right.$, where $f_{g}(\cdot)$ is the fitness value of the optimal individual in the current generation of population). If one of the above conditions is met, then evolution should be terminated, and the optimal individuals in the current generation of population will be output as the optimal segments; if the above condition is not satisfied, differential evolution should be continued.

\section{(4) mutation}

Differential evolution realizes individual mutation through difference strategy. In another word, the mutation vector is gained by adding the base vector (current vector) and difference vector after factor zoom. Different base vector selection modes and different numbers of difference vectors will constitute different mutation operators. The mutation operator is the most important operator of 
differential evolution algorithm. At present, DE researchers have designed some common mutation operators. In this study, the optimal individual in the current generation of population will be used as base vector, and the number of difference vectors is 1 . They can be expressed with Equation 4 .

$$
\mathrm{V}_{i}(g)=x_{\text {best }}(g)+F\left(x_{r 1}(g)-x_{r 2}(g)\right)
$$

Where $x_{\text {best }}(g)$ is the optimal individual vector in the current population; $x_{\mathrm{i}}(g)$ is the target (father) vector; $r 1 \neq r 2 \neq i$ are two different integers randomly selected in the set $\{1,2, \ldots, N P\}$; $V_{i}(g)$ is the mutation vector; $F$ is the zoom factor or mutation probability, with the value range of $[0,1+]$.

\section{(5) repair}

For some special problems, vector individuals after mutation might fall outside the search space. At this time, repair operation should be adopted. See Equation 5 for repair operation.

$$
V_{i, j}(g)=\left\{\begin{array}{l}
\min \left\{U_{j}, 2 L_{j}-V_{i, j}(g)\right\}, \text { if } V_{i, j}(g)<L_{j} \\
\max \left\{L_{j}, 2 U_{j}-V_{i, j}(g)\right\}, \text { if } \mathrm{V}_{i, j}(g)>U_{j}
\end{array}\right.
$$

\section{(6) crossover}

Differential evolution algorithm generates test vector via crossover which is conducted through base and mutation vector. Besides, discrete crossover is used to enhance diversity of the population. Common crossovers include binomial crossover and exponential crossover. Exponential crossover is adopted in this study. The crossover is carried out according to Equation 6.

$$
\mathrm{u}_{i, j}(g)=\left\{\begin{array}{l}
v_{i, j}(g), j=\langle l\rangle_{D},\langle l+1\rangle_{D}, \ldots,\langle l+L+1\rangle_{D} \\
x_{i, j}(g), \quad \text { else }
\end{array}\right.
$$

Where $\langle l\rangle_{D}$ indicates modulus operation of D, $l$ is any random integer in [1, D], and the integer $L$ is between 1 and $\mathrm{D}$.

\section{(7) Selection}

DE adopts greedy selection strategy and selects the optimal individual according to the fitness value of target vector and test vector. The selection operation is shown in Equation 7.

$$
x_{i}(g+1)=\left\{\begin{array}{l}
u_{i}(g), f\left(u_{i}(g)\right)<\mathrm{f}\left(x_{i}(g)\right) \\
x_{i}(g), \text { else }
\end{array}\right.
$$

According to explanation about selection of the optimal parameters via differential evolution algorithm, this method covers 5 parameters which are population size $N P$, maximum generation of differential evolution $G$, difference strategy, zoom factor $F$ and crossover probability $C R$. In which $N P, F$ and $C R$ are control parameters. These control parameters will affect the search for the optimal parameters and convergence rate. High population will increase diversity of individuals and improve possibility of searching the optimal solution, but it will reduce convergence rate. Low population can accelerate convergence, but local convergence might be caused easily or the evolution can stop. The zoom factor $F$ is used to control the search step. If the value of $F$ is small, convergence can be accelerated, but local convergence will be caused easily and "precocity" phenomenon might occur. If the value of $F$ is large, the possibility for the algorithm to jump out of local optimal solution will be increased. But the convergence rate might be reduced if $F>1$. According to the suggestions of Storn and Prince [11], the reasonable value of $F$ is in the scope of $[0.4,1.0]$. The suitable initial value should be $F=0.5$. Therefore, this value is taken for zoom factor in this study. Setting of the crossover probability $C R$ mainly depends on the problems. As for problems with independent variables, small value can be taken for $C R$; in terms of problems with interdependent variables, large value should be taken for $C R$. And the initial value of $C R$ sets to 0.3 is suitable, so this value is taken in this study. Therefore, when differential evolution algorithm is used to select the optimal parameters of image segmentation, we just need to further set the population size $N P$, the maximum generation of differential evolution $G$ and threshold value of different degree termination $t h_{f}$. 


\section{Results and analysis}

The two subsets of aerial remote sensing image captured by DMC sensor with three bands [i.e., red, green and blue] are used for experiment. The two subset images are all 400*400 pixels size. Figure 3 and Figure 4 are the two subsets images and the reference polygons. In Figure 3, we used three parking lots and two playgrounds as reference objects. In Figure 4, we used one road, two garden plots and one pond as reference objects.
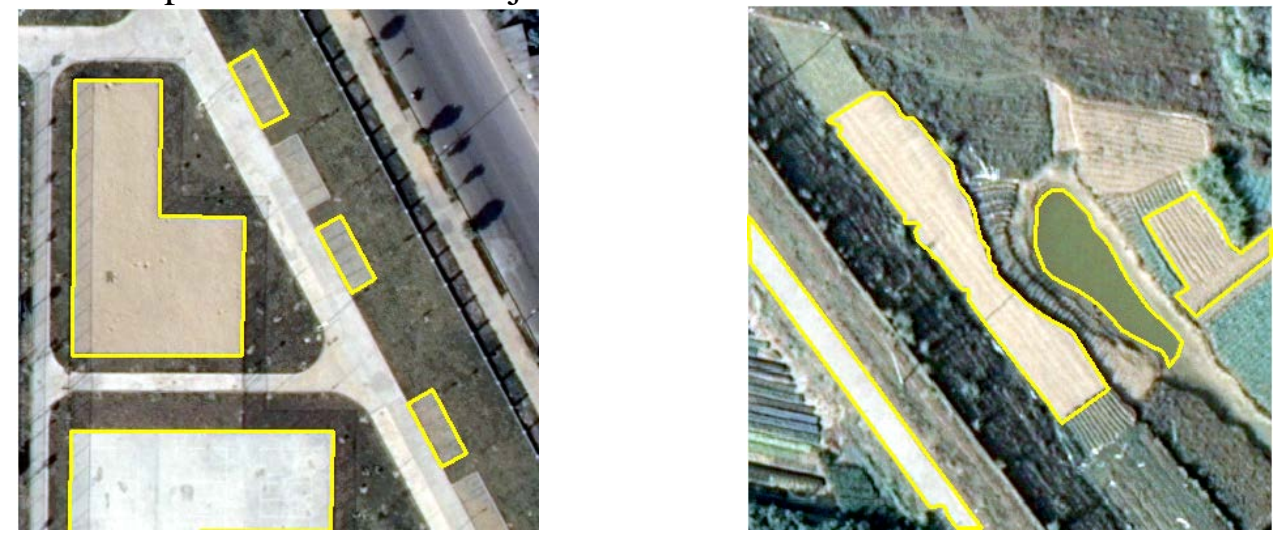

Fig.3. Image subset1 and its reference polygons Fig.4. Image subset2 and its reference polygons

For the experiment, the differential evolution optimization algorithm parameters were set as follow: the population was 100 , generation was 5 and minimum error was $1 \mathrm{E}-15$ (the other parameters were default: CR was 0.3 , F was 0.5 and used exponential crossover). The searching range of the region growing segmentation parameters were set as follow: the range of scale parameter was $[10,150]$, the weight of color was $[0,1]$, the weight of compactness in the shape feature was $[0,1]$.
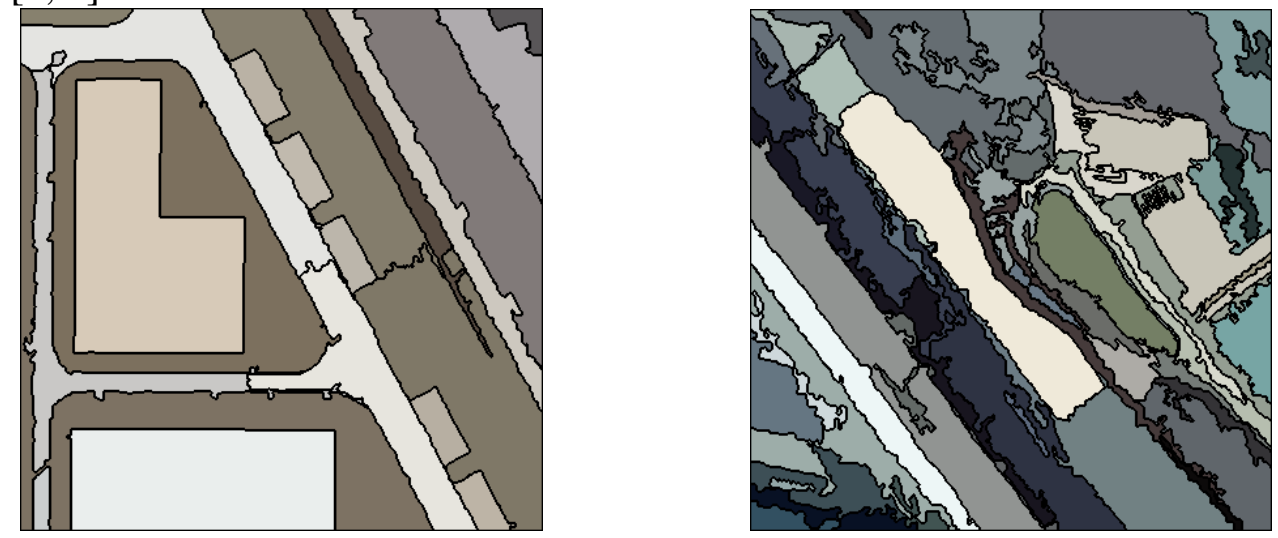

Fig.5. The segmentation result of mage subset1

Fig.6. The segmentation result of mage subset2

The segments of subset1 obtained by region growing segmentation algorithm using the optimal parameters obtained by differential evolution optimization algorithm shows in Figure 5 and the segments of subset2 shows in Figure 6. The optimal parameters and the fitness values of the two image subsets obtained by differential evolution are list in Table 1.

Table.1. The optimal parameters and the fitness obtained by differential evolution

\begin{tabular}{ccccc}
\hline \multirow{2}{*}{ image } & \multicolumn{3}{c}{ Parameters of region growing segmentation } & \multirow{2}{*}{ Fitness } \\
& scale & Weight of color & Weight of compactness & \\
\hline Subset1 & 76 & 0.100 & 0.346 & 0.0042 \\
Subset2 & 98 & 0.680 & 1.000 & 0.0096 \\
\hline
\end{tabular}

The Figure 5 shows that: the five parking lots align to the road were well segmented; the two playgrounds were also well segmented; most of the other coarse objects on the image were also segmented well at this scale; and the fine objects, such as trees and shadows of trees were not segmentation; the parameters are suitable for segmenting coarse objects. The segments based on the optimal parameters of the subset2 showed in Figure 6, the road was segmented correctly except that the boundary was not neat because of the road border was covered by some shrubs; the middle garden plot and the pond were segmented well; the most part of right garden plot object was segmented correctly except that the right upper part was merged with the upper garden plot. 


\section{Conclusion}

This paper designed a method to automatically optimize the parameters of region growing algorithm based on the differential evolution algorithm. Because of Differential evolution algorithm is a heuristic optimization method, use this method to select the parameters for optimal segments is objective and efficient. The experiment results of the two subset aerial remote sensing image showed that use this method to select the optimal segmentation parameters can get the accuracy result and improve the efficiency.

\section{References}

[1]. Baatz M, Schape A. Multiresolution segmentation: an optimization approach for high quality multi-scale image segmentation[J]. Journal of Photogrammetry and Remote Sensing, 2000, 58(3-4)..

[2] Hay G J, Blaschke T, Marceau D J, et al. A comparison of three image-object methods for the multiscale analysis of landscape structure[J]. ISPRS Journal of Photogrammetry and Remote Sensing, 2003, 57(5): 327-345.

[3] Meinel G, Neubert M. A comparison of segmentation programs for high resolution remote sensing data[J]. International Archives of Photogrammetry and Remote Sensing, 2004, 35(Part B): 1097-1105.

[4] Yujin Zhang. Image engineering-middle-image analysis [M], Tsinghua press,2012.8.

[5] Espindola G, Camara G, Reis I, et al. Parameter selection for region - growing image segmentation algorithms using spatial autocorrelation[J]. International Journal of Remote Sensing, 2006, 27(14): 3035-3040.

[6] Johnson B, Xie Z X. Unsupervised image segmentation evaluation and refinement using a multi-scale approach[J]. Isprs Journal of Photogrammetry and Remote Sensing, 2011, 66(4): 473-483.

[7] Drăguţ L, Eisank C. Automated object-based classification of topography from SRTM data[J]. Geomorphology, 2012, 141-142: 21-33.

[8] Drăguţ L, Csillik O, Eisank C, et al. Automated parameterisation for multi-scale image segmentation on multiple layers[J]. ISPRS Journal of Photogrammetry and Remote Sensing, 2014, 88: $119-127$.

[9] Liu Y, Bian L, Meng Y, et al. Discrepancy measures for selecting optimal combination of parameter values in object-based image analysis[J]. Isprs Journal of Photogrammetry and Remote Sensing, 2012, 68: 144-156.

[10] Witharana C, Civco D L. Optimizing multi-resolution segmentation scale using empirical methods: Exploring the sensitivity of the supervised discrepancy measure Euclidean distance 2 (ED2)[J]. Isprs Journal of Photogrammetry and Remote Sensing, 2014, 87: 108-121.

[11] Storn R, Price K. Differential evolution - A simple and efficient heuristic for global optimization over continuous spaces[J]. Journal of Global Optimization, 1997, 11(4): 341-359.

[12] Pont-Tuset J, Marques F. Measures and Meta-Measures for the Supervised Evaluation of Image Segmentation[C]. IEEE Conference on Computer Vision and Pattern Recognition- CVPR, 2013: 2131-2138. 